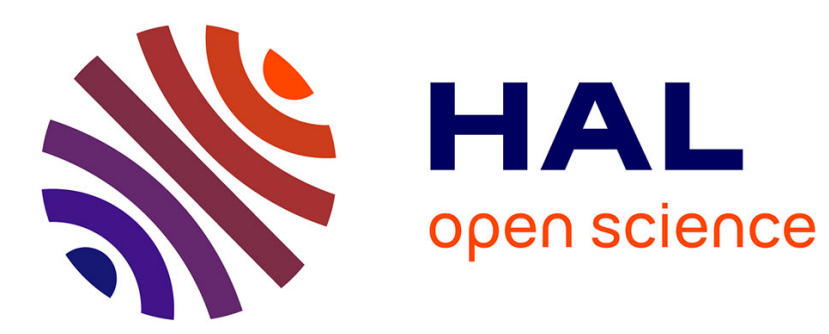

\title{
Seamless multicast : An SDN-based architecture for continuous audiovisual transport
}

Constant Colombo, Francis Lepage, René Kopp, Eric Gnaedinger

\section{To cite this version:}

Constant Colombo, Francis Lepage, René Kopp, Eric Gnaedinger. Seamless multicast: An SDNbased architecture for continuous audiovisual transport. Telecommunication Systems, 2021, 78 (2), pp.187-202. 10.1007/s11235-021-00796-9 . hal-03334404

\section{HAL Id: hal-03334404 \\ https://hal.science/hal-03334404}

Submitted on 3 Sep 2021

HAL is a multi-disciplinary open access archive for the deposit and dissemination of scientific research documents, whether they are published or not. The documents may come from teaching and research institutions in France or abroad, or from public or private research centers.
L'archive ouverte pluridisciplinaire HAL, est destinée au dépôt et à la diffusion de documents scientifiques de niveau recherche, publiés ou non, émanant des établissements d'enseignement et de recherche français ou étrangers, des laboratoires publics ou privés. 


\title{
Seamless Multicast : an SDN-based architecture for continuous audiovisual transport
}

\author{
Constant Colombo (Corresponding Author) - Francis Lepage • \\ René Kopp · Eric Gnaedinger
}

Received: date / Accepted: date

\begin{abstract}
For audiovisual network operators, end-users satisfaction is a major issue. This is the case for TDF who operates a nationwide network in France whose main purpose is to carry Digital Terrestrial Television (DTT) streams. Such audiovisual content is forwarded through multicast real-time streams which require continuity of service. Therefore, the main goal of this work is to define a new architecture to prevent impact during network healing time. The proposed architecture aims to use a pair of redundant multicast trees, and ensure their seamless resiliency. This architecture called "Seamless Multicast" takes advantage of the networkend equipment's ability to receive and combine two identical streams, complete or not. The main contribution of this paper is the development and evaluation of an algorithm for the computation of a pair of multicast trees and the associated hitless deployment scheme. Implementation requires an Software-Defined Networking (SDN) architecture, in which performance knowledge and bandwidth management are centralized in a controller. A proof of concept controller has been used for validation of the architecture's global behaviour using a virtualized environment in multiple scenarios.
\end{abstract}

Keywords Multicast, Seamless, Real-time audiovisual stream, Disjoint trees, SDN, OpenFlow.

C. Colombo, F. Lepage

CRAN \& Université de Lorraine, France

Tel.: +33645616534

E-mail: constant.colombo@univ-lorraine.fr

R. Kopp

TDF SAS - 1 Rue Marconi, 57070 Metz, France

Tel.: +33387207543

E-mail: rene.kopp@tdf.fr

E. Gnaedinger

Université de Lorraine, France

\section{Acronyms}

DMDT Delay-constrained Minimal Destination maximally-disjoint Trees. 4,5

IS Iterative SHERPA. 2, 6-8, 10, 13

PCE Path Computation Element. 10

SDN Software-Defined Networking. 1, 2, 9, 10, 13

SHERPA SHaring-Edges Restrained PAths. 6, 7

\section{Introduction}

Availability and continuity of service are two of the main problematics addressed by network operators, as they directly impact the end-user degree of satisfaction. Availability is defined as the global uptime of a system over a period, while service continuity describes the uninterrupted uptime of a service.

TDF is a French network operator, whose main activity is to provide a transport infrastructure for realtime audio and video streams nationwide, specifically Digital Terrestrial Television channels (DTT) and national radios, from the recording studios to broadcasting antennas. TDF operates its own network which is disconnected from the Internet.

DTT streams are real-time, which implies not only that transport must be fast and stable, but also that data re-transmission is impossible. As a consequence, TCP-associated methods cannot be applied, and any failure on the network may create content impact. Enduser satisfaction can be studied using Quality of Experience (QoE) [1] metrics, and addressed through Quality of Service (QoS) [2] tools. Usually, commitments on availability, latency, jitter, and loss are specified for each business customer in the Service Level Agreement (SLA). 

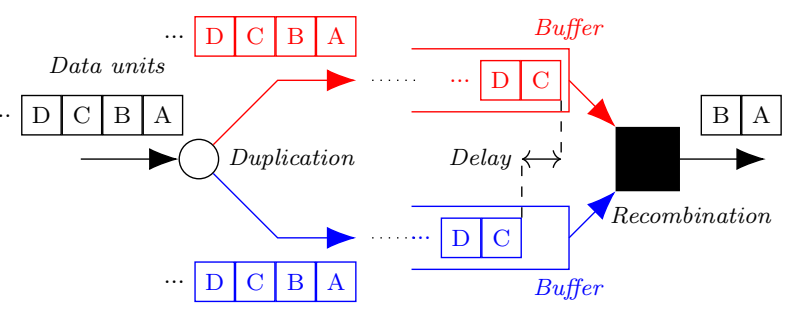

Fig. 1 Seamless Reception concept

Based on Seamless Reception techniques, this works' goal is to exploit redundancy and resiliency to propose a new reliable transport architecture for continuous service. Seamless Reception is the set of techniques enabling packet selection and recombination from multiple identical data streams to provide an output without any apparent interruption, as illustrated in Fig. 1. The standard Seamless Protection Switching (SPS) [3] defines the combination process of two redundant streams into a single coherent stream, without any cut in case of data loss in the streams. Such techniques help enforce the continuity of service commitment specified in the SLA, in case of degradation or failure of one of the stream.

In this paper, Seamless Switching advantages are the basis for the proposed architecture, called Seamless Multicast. This architecture is bipartite : an novel algorithm is used to compute two redundant trees from the source of the stream to the set of destinations, and a novel deployment scheme ensures the dynamic hitless implementation of those multicast trees in a network.

The principle is the following. A pair of multicast transport tree is computed, both of them deployed as active, carrying the same data to the same set of destinations. Each destination receives the streams through Seamless Reception. Therefore, if only one of the stream is impacted by a failure, the destination still receives all the data. As such, the main computational objective is to find the pair of most independent trees. It can be easily demonstrated that the probability of simultaneous failure of both trees is minimal if both trees are independent. This scheme differs from backup mechanisms, as both trees are active at the same time and supply the same destinations. A major drawback lies in overall network bandwidth consumption, as twice more bandwidth is required for the same service due to data duplication. That is why the second main computational goal is to minimize the total bandwidth consumption.

While redundancy ensures a proactive protection in case of a simple failure, some destinations may not be protected whenever multiple failures occur. Similarly, when any failing element resumes functionning, the deployed multicast trees are not as optimal as they could

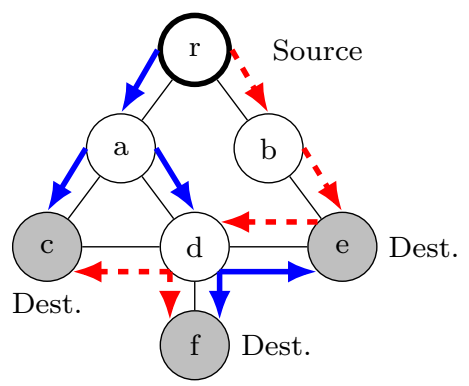

Fig. 2 Maximally independent delay-constrained trees

be. That is why the Seamless Multicast architecture involves dynamic deployment. Furthermore, during any deployment, no impact should be visible. The scheme proposed in this paper enables hitless deployment in case of network component failure or recovery.

To summarize, the Seamless Multicast architecture not only builds the lightest pair of maximally independent redundant trees from the source of the stream to the destinations as illustrated in Fig. 2, but also ensures hitless dynamicity of those trees to account for multiple failures.

However, in order to implement and test the proposed architecture, traditional networking protocols are not adaptable enough. This paper also presents an actual implementation of the architecture through to SDN technologies.

Software-Defined Networking (SDN) [4] is a network paradigm in which the control plane and the data plane are physically isolated. An entity called "controller" handles routing and switching decisions while network nodes only perform data forwarding. Thanks to network programmability, any functionality can be implemented in a centralized fashion. This paper's SDN-based solution is illustrated in Fig. 3. The network is divided in four main planes : the application plane, describing service in a non-technical fashion, usually specifying an interface for the network operator ; the control plane, carried by the controller, where this paper's contribution resides ; the management plane, responsible for network management; the data plane, which applies the controller's traffic forwarding decisions.

This paper presents the complete Seamless Multicast architecture and experimental validation. It is organized as follow: Section 2 presents the state of the art techniques for multicast reliable communications. In Section 3 industrial constraints are described and transposed into a new graph theory formulation. Related works on this specific multi-tree computation problem are reviewed. In Section 4, the proposed algorithm Iterative SHERPA (IS) is explained. Section 5 presents the novel dynamic scheme for trees deployment. The Seamless Multicast implementation using SDN is detailed 


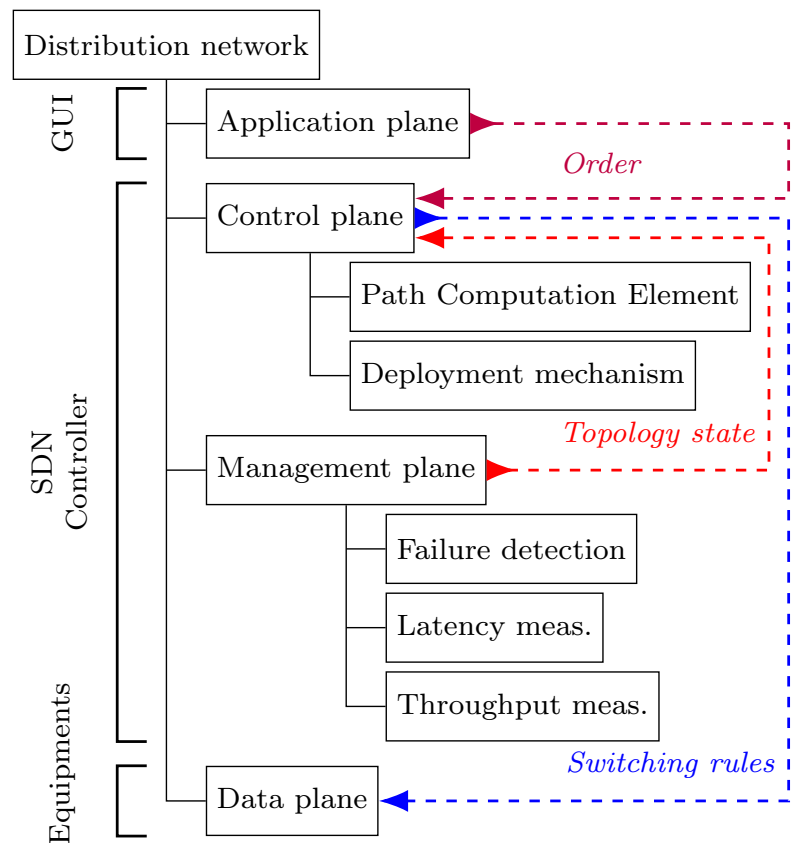

Fig. 3 Architecture Overview

and evaluated in Section 6. Experimentation shows that the architecture does provide continuity of service. Finally, Section 7 uses theoretical values to show the Seamless Multicast architecture also improves availability compared to the MVPN standard.

\section{Related works on reliable multicast transport}

Solutions for reliable transport have been studied in the scientific literature and industry for various network architectures.

Interior Gateway Procotol (IGP), in other words rerouting, is the base of most traditional IP solutions, through dynamic computation or backup paths. The industrial standard Multicast VPN (MVPN) [5] is a combination of the IGMP and PIM protocols, both relying on the IGP to establish a multicast tree and to ensure its resiliency. Rerouting-based resiliency schemes necessarily cause micro-cuts of the service, and thus impact the content viewing. Usually, buffers of the receiving equipment are designed to absorb a part of the rerouting time, but end equipment's' buffers are limited due to the real-time nature of the streams. As a consequence, the only solution would be to improve rerouting performance, which can be complex and costly.

Within the same industrial context as this paper, the method proposed [6] by Pirlot et al. develops robustness through network survivability, and provides modeling tools for MVPN solutions to evaluate the protocol's reliability. Their works propose enhanced MVPN variants showing that improvement of a traditional architecture is possible but limited due to the aforementioned resiliency impact.

In a different context, Xiong and Chen propose a scheme for data-center environment based on multiple trees to ensure reliable multicast distribution [7]. This trees are specifically computed for hierarchical network. Using rapid path rerouting through pre-computed multicast trees, loss is avoided. Consecutive network events are not considered, and impact cannot be avoided due to the computation time.

To ensure communication reliability over Networked Controlled Systems, a genetic algorithm to deploy two spanning trees is proposed by Kubler et al. [8]. Though it ensures redundancy and protects the network thanks to redundancy, it is bandwidth-inefficient. In audiovisual networks, bandwidth consumption is a major issue.

Different SDN-based solutions can be found in the literature. A proactive backup path deployment scheme is proposed by Ghannami and Shao [9]. While this solution improves service availability, it is not based on multicast trees and is not bandwidth-efficient.

A reliable unicast solution is proposed by Vafaei et al. in [10] for audiovisual streams. Similar QoS constraints are applied to a redundant path computation problem. The proposed Ant Colony algorithm is intended for Vehicular Networks (VANET), dynamic by nature, and tends to the real-time constraint through rapid rerouting. In these works, the independent path do not carry the same data, but two parts of the stream. As such, the bandwidth optimization goal is not considered.

A survey by Islam et al. [11] highlights the challenges of the SDN-based multicast architectures, notably QoS and scalability, both in tree computation and node forwarding states. Other issues linked to the dynamic nature of some multicast uses, or distribution's security are also considered. This survey shows that SDN-based multicast proposal often aim to solve the customer subscription issue, which is not relevant in our case because the network operator TDF selects the source and destination of a service per customer's contract. The survey concludes that SDN approaches do not propose a general framework for multicast applications, but will enable the development of solutions for specific environments. In these works, we focused on architectures aiming to improve continuity of service under various constraints.

In [12] Yen et al. propose an hybrid multicast solution. It integrates SDN architecture to traditional IP architecture using PIM. Though these works do not provide novel routing methods for reliable multicast, 
its goal is to interconnect the industry legacy standard to new solutions.

Most of the reliable multicast SDN-based solutions are either proactive or reactive. An architecture exploiting cached multicast trees for fast deployment is proposed by Kotani et al. in [13]. Similarly, Renganathan et al. develop a subtree based approach, with precomputed segment reroutes in [14].

The works of Mohammadi et al. [15] are very close to this paper's context : the objective is to exploit SDN technologies to provide reliable and optimized multicast transport solutions. The formulation of the Delay Constraint Least Cost tree problem (DCLC) involves similar constraints and optimization goal. The proposed approach uses a single multicast tree, and reactive rerouting. It showed greater performance than traditional IP solutions in terms of latency, jitter and packet loss, but continuity of service has not been studied in [15].

The novelty of the proposal described in this paper is to approach absolute continuity of service by using both proactivity and reactivity. Seamless receivers are powered by multicast trees that are as independent as possible, under constraint delay, limiting bandwidth consumption and ensuring seamless redeployment in the event of a tree failure.

\section{Audiovisual transport graph theory formulation}

Audiovisual transport is associated with specific characteristics, and the first novel contribution of this work is to propose a formal definition of the addressed issue.

\subsection{Problem statement}

Multicast is a communication mode in which data are sent to a group of receivers in a single transmission using data duplication on the transport path. This differs from multiple unicast transmissions, that is to say data duplication at source level. As such, multicast communications rely on distribution trees. If network topology is modelled as a graph, tree computation methods from graph theory can be applied. A simple example is the spanning tree, a multicast connection to every node, efficient enough in simple topologies and computable using well-known algorithms.

This work was realized in collaboration with the network operator TDF, and the problem's constraints are induced by the context. The data to be transported form a continuous real-time stream. As a consequence, it must be carried without loss and within minimal delay.
Lossless transportation, in other words continuity of service, is the issue addressed by this paper's proposal. The concept is for each destination to be reached through two disjoint paths. The two paths carry identical streams, providing path diversity for Seamless Reception techniques. The complete set of paths forms a multicast tree, in which pair of paths must be independent. Given real network topologies, totally disjoint paths are not always possible. For example, some areas may be reachable by a unique path for geographic reasons. This means a Single Point of Failure is unavoidable in some areas of the network. Therefore, pathdisjointedness is not required to be strict in the problem statement.

The minimal-delay constraint is a global service limitation : from the source to any destination, a delay threshold value must be respected. This constraint is usually specified in the SLA, and emerges from the realtime nature of the data. In practice, each edge of the graph will be set with the maximum link delay measured over a significant period, to ensure that the delay threshold will not be violated over the real network because of jitter.

Finally, as the network carries multiple services for different customers, bandwidth management must be considered. A minimum of total bandwidth must be consumed per service. This constraint is one of the reasons for using only two multicast trees for path diversity. While more redundancy would improve the system, the bandwidth cost is too important. In practice, prior to any computation, links that have insufficient available bandwidth are removed from the usable topology.

Given the goal and constraints, the problem is stated as finding Delay-constrained Minimal Destination maximally-disjoint Trees (DMDT).

\subsection{DMDT proposed cost function}

In a directed graph $G_{0}=(V, E)$, with $V$ the set of vertices and $E \in V^{2}$ the set of edges, weighted by $C_{0}$ and $D$ respectively link-cost and link-delay, given a delay constraint $\Delta$, the DMDT problem's goal is to find for a source $s$ and a destination set $T$ a pair of trees $A_{R}$ and $A_{B}$ connecting $s$ to $T$, maximally-disjoint per destination and globally lightest.

Let $R$ and $B$ denote the two binary vectors corresponding to the trees $A_{R}$ and $A_{B}$. Each element of those vectors represents a link $(x, y) \in E$. Their value is 1 if the link belongs to the tree, 0 otherwise. Let $Z$ denote a vector which elements represent the usage of links in both $A_{R}$ and $A_{B}$ to reach the same destination. Values of $Z(x, y)$ range from 0 to $|T|$, because each time a link 
is used by both trees to reach a destination, $Z(x, y)$ is increased by 1 .

The cost function (1) is composed of three terms. The first two are the sum of each tree's weight, and the third one represents the cost of inter-dependence between the trees.

$$
\begin{aligned}
\mathcal{F}(R, B)= & \sum_{(x, y) \in E} C_{0}(x, y) R(x, y) \\
& +\sum_{(x, y) \in E} C_{0}(x, y) B(x, y) \\
& +2 \sum_{(x, y) \in E} C_{0}(x, y) \sum_{(x, y) \in E} Z(x, y)
\end{aligned}
$$

Using twice the total weight of the graph as a coefficient gives a greater importance to independence. The cost optimization becomes secondary. This is proven in Theorem 1.

Theorem 1 Let $\beta_{R B}$ denote the sum of $Z$ values for a given pair of trees $A_{R}, A_{B}$.

$\forall A_{1}, A_{2} \subset E$ and associated $\beta_{12} \geq 0$,

$\forall A_{3}, A_{4} \subset E$ and associated $\beta_{34} \geq 0$,

If $\beta_{12}<\beta_{34}$ then:

$$
\begin{aligned}
& C_{0}\left(A_{1}\right)+C_{0}\left(A_{2}\right)+\beta_{12} * 2 * C_{0}(E) \\
< & C_{0}\left(A_{3}\right)+C_{0}\left(A_{4}\right)+\beta_{34} * 2 * C_{0}(E)
\end{aligned}
$$

Proof.

Given $G_{0}=(V, E)$ a graph weighted by $C_{0} \geq 0$,

$\forall A \subset E, A \neq \emptyset$,

$0<C_{0}(A) \leq C_{0}(E)$

$\Rightarrow 0<C_{0}\left(A_{i}\right)+C_{0}\left(A_{j}\right) \leq 2 C_{0}(E), \forall A_{i}, A_{j} \subset(E)$

$\forall A_{1}, A_{2} \subset E$ and associated $\beta_{12} \in \mathbb{N}^{+}, A_{1} \neq \emptyset, A_{2} \neq \emptyset$, $\forall A_{3}, A_{4} \subset E$ and associated $\beta_{34} \in \mathbb{N}^{+}, A_{3} \neq \emptyset, A_{4} \neq \emptyset$,

$0 \leq \beta_{12}<\beta_{34}$

$\Rightarrow \beta_{12}+1 \leq \beta_{34}$

as $\beta_{12}, \beta_{34} \in \mathbb{N}^{+}$

$\Rightarrow \beta_{12} * 2 C_{0}(E)+2 * C_{0}(E) \leq \beta_{34} * 2 C_{0}(E)$

as $C_{0}(E)>0$

$$
\begin{aligned}
& \Rightarrow \quad \beta_{12} * 2 C_{0}(E)+C_{0}\left(A_{1}\right)+C_{0}\left(A_{2}\right) \\
& \leq \beta_{34} * 2 C_{0}(E) \text { according to }(3) \\
& \Rightarrow \quad \beta_{12} * 2 C_{0}(E)+C_{0}\left(A_{1}\right)+C_{0}\left(A_{2}\right) \\
& <\beta_{34} * 2 C_{0}(E)+C_{0}\left(A_{3}\right)+C_{0}\left(A_{4}\right) \\
& \text { as } C_{0}\left(A_{3}\right)>0 \text { and } C_{0}\left(A_{4}\right)>0
\end{aligned}
$$

This proposed formulation of the cost function does not guarantee the absence of cycles nor multiple paths to a single node. Nevertheless, the minimization of the function eliminates this issue, as pseudo-trees with cycles or multiple path will have a higher cost. The optimal solution does not contain any by definition.

\section{$3.3 \mathcal{N} \mathcal{P}$-hardness}

The DMDT problem is $\mathcal{N} \mathcal{P}$-hard and this section focuses on its proof.

\section{Theorem 2}

The DMDT problem is $\mathcal{N} \mathcal{P}$-hard.

\section{Proof.}

The MDP problem of finding Maximally-independent Delay-constrained Paths is $\mathcal{N} \mathcal{P}$-hard as proven by Orda and Sprintson [16].

The MDP problem is a special case of the DMDT problem. Using the following DMDT definition: find a minimal pair of redundant trees connecting a root node to $|T|$ destinations, delay-constrained, in which internal paths to a destination are maximally-independent ; for $|T|=1$, the DMDT problem becomes an instance of the MDP problem.

By generalization of the MDP problem which is $\mathcal{N} \mathcal{P}$-hard, the DMDT problem is itself $\mathcal{N} \mathcal{P}$-hard.

In practice, $\mathcal{N} \mathcal{P}$-hardness means that it is impossible to guarantee finding an optimal solution fast. In other words, either a solution is found quickly with the hope that it is satisfying for the application, or the computation of an optimum is fast enough to match the application constraints. In this paper, we propose a heuristic to solve the DMDT problem meeting the computation time constraint of the architecture's deployment mechanism presented in Section 5.

\subsection{Related works on DMDT computation}

In order to solve the DMDT problem, literature provides no method as far as we know. Nevertheless, to compute a single delay-constrained multicast tree, many heuristics have been proposed such as by Forsati et al. [17], and even an exact solution by Feng [18].

Medard et al. proposed an algorithm to compute redundant trees in an edge-redundant graph [19], that is to say when it is guaranteed that two completely independent paths can be found between any pair of vertices in the graph. The proposed algorithm can account for costs and a delay constraint. However, this work relies 
on undirected graphs, while within our context we consider different costs on each direction of a link, as audiovisual content-providing services are unidirectional. Furthermore, the edge-redundant graph requirement is not met in practice, and thus the algorithm cannot be used.

Bejerano et al. intended to provide another algorithm [20-22]. This method relies on directed graphs to result in close to optimal solutions, but does not consider any delay constraint and requires graphs with too specific properties.

\section{Architecture's dedicated routing algorithm}

In order to solve the DMDT problem, the proposed heuristic uses a delay-constrained shortest path algorithm (CBF) from the literature [23], and a newly proposed delay-constrained shortest pair of path algorithm (SHERPA).

\subsection{Delay-constrained shortest path algorithm}

For this work, the Constrained Bellman-Ford algorithm (CBF) [23] has been selected. It is based on the BellmanFord algorithm [24].

The CBF algorithm is a breadth-first search, discovering paths with increasing delay while recording the shortest path to each node visited, until the exploration cumulative delay exceeds the delay constraint.

\subsection{SHaring-Edges Restrained PAths - SHERPA}

In a graph $G_{0}=(V, E)$, for a given pair $(s, t) \in V \times V$, the SHaring-Edges Restrained PAths (SHERPA) [25] algorithm's goal is to find two lightest delay-constrained maximally edge-disjoint paths from $s$ to $t$. It is based on the Suurballe-Tarjan algorithm [26], which does not account for delay constraint and ensures only strict disjointedness. To create the proposed algorithm, it has been altered in two ways. Firstly, the shortest path algorithm used (Dijkstra's algorithm) has been replaced by the delay-constrained algorithm CBF. The second modification consists in a re-weighting scheme imposing a maximal weight on some links to provide relaxed disjointedness.

In the original algorithm, links that belonged to the first computed path were deleted from the graph in order to compute the second independently. Instead, in our proposed variant, such links are only made prohibitive to use for a second path computation. Any other link is weighted following the Suurballe-Tarjan scheme, that is to say subtracting from their original cost the difference of distance from the source of their ending point and starting point.

For a directed graph $G_{0}=(V, E)$ weighted by $C_{0}$, let $d(v)$ denote the cost in $C_{0}$ of the shortest path from $v \in V$ to $s$. Let $P_{a}$ denote the set of links of the first computed delay-constrained path, whose weight is to be made prohibitive. The new graph cost $C_{1}$ of $G_{0}$ is defined by equation (4) $\forall(x, y) \in E$ :

$C_{1}(x, y)= \begin{cases}\sum_{e}^{e \in E} C_{0}(e) & \text { if }(x, y) \in P_{a} \\ C_{0}(x, y)-d(y)+d(x) & \text { otherwise }\end{cases}$

For a directed graph $G_{0}=(V, E)$ weighted by $C_{0}$ and $D$ respectively cost and delay, a source $s$, a destination $t$ and a delay constraint $\Delta$, the SHERPA algorithm can be summarized as follows:

1. A first delay-constrained shortest path $P_{a}$ is computed from $s$ to $t$;

2. The graph is re-weighted following equation (4);

3. A second delay-constrained shortest path $P_{b}$ is computed from $s$ to $t$;

4. $P_{a} \cup P_{b}$ contains the two paths after sort out operations.

The complete procedure is detailed in Appendix A, Algorithm 1 and it is illustrated in Fig. 4. In the example presented in Fig 4a, the goal is to connect the node $s$ to the node $t$, with two maximally-independent paths under the delay constraint of 5s. Every link has a delay of 1 s except $d-c$. Fig 4 b illustrates the first path $P_{R}$ computed using CBF. The graph is re-weighted in Fig $4 c$ according to the SHERPA algorithm. The most notable element is the prohibitive weight of the links $s-b, b-c$ and $c-t$, which were part of the path $P_{R}$. The second path $P_{B}$ is computed by CBF and illustrated in Fig 4d. The two paths are as independent as possible, while not using the link $d-c$, which would provide more independence but would also violate the delay constraint.

Based on the Suurballe-Tarjan algorithm's variation for node-disjointedness, our proposed algorithm SHERPA is adjustable to find delay-constrained maximally node-disjoint paths. This variation can be used in higher level algorithms aiming to find node-disjoint trees.

The SHERPA algorithm allows to find a pair of paths, and is used in the novel IS algorithm presented below.

\subsection{Iterative SHERPA - IS}

The proposed algorithm to solve the DMDT problem is based on the multipath algorithm SHERPA. It is 


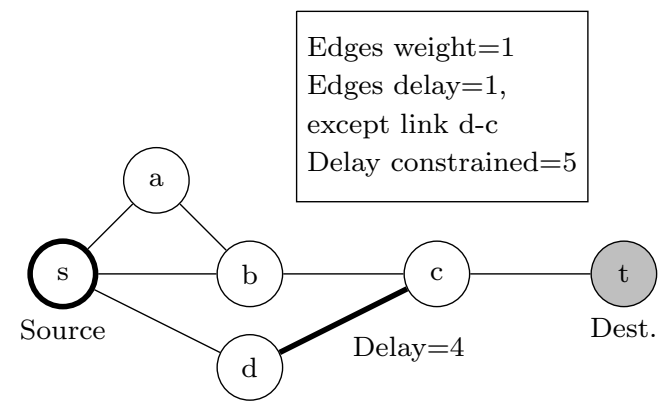

(a) Base graph

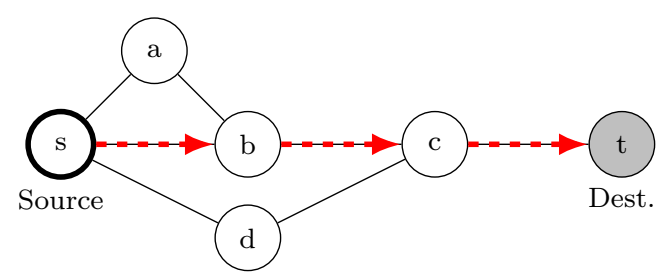

(b) Path $P_{R}$

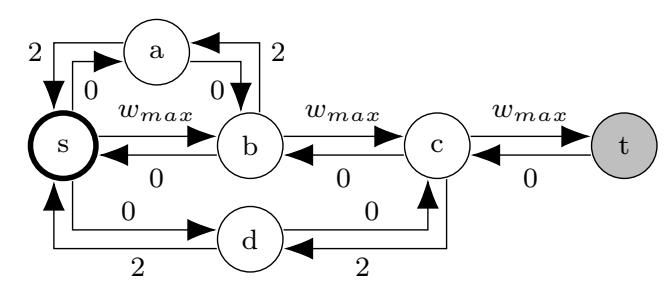

(c) Reweighting

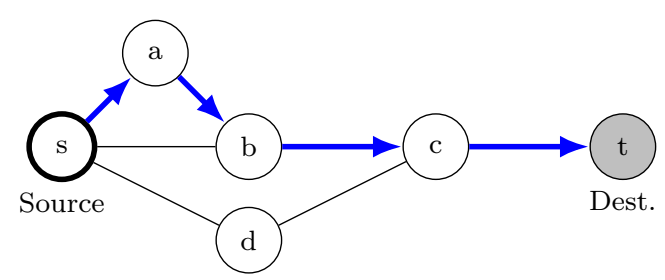

(d) Path $P_{B}$

Fig. 4 SHERPA illustration

called Iterative SHERPA (IS) [27]. IS iteratively computes pairs of paths using SHERPA, and assigns them to the trees according to the maximum independence provided.

The IS procedure relies on iterations of the SHERPA algorithm. An instance of the SHERPA algorithm runs for each destination not yet reached by the trees to provide a pair of paths starting at the trees' root (i.e. the source node). Then, in increasing pair-cost order, each path of a pair is assigned to one of the trees so that independence is maximal between the newly completed trees. If the provided independence is equal for both possible affectations, no decision is made and the affected node is skipped to be processed in later iterations. When all nodes have been processed, if all remaining nodes cannot be assigned, an arbitrary decision is made and the algorithm resumes. This process is repeated until all destinations are reached by the trees.

Destinations are processed in increasing pair-cost order, so that the algorithm can be deterministic. Better solutions could be found using a different order, but determinism is a major issue for practical use and network management.

Node-disjointedness can be achieved by using the node-disjoint variation of SHERPA algorithm, and by estimating the best path assignation using the number of common vertices instead of common edges.

The proposed IS scheme ensures maximal path diversity per destination. Path cost is minimized, but total tree cost is not guaranteed to be minimized, as path affectation only depends on independence. The complete procedure is detailed in Appendix A, Algorithm 2. The algorithm is illustrated in Fig. 5. Original graph representation and constraints are described in Fig. 5a. The IS algorithm builds a solution using node $r$ as source, and $a, d, e$ as destinations. According to the algorithm, the pairs of paths computed are illustrated in Fig. 5b, 5d and 5f, and assigned respectively in Fig. 5c, 5e and 5g.

\subsection{Validity of the solutions}

The process of the IS algorithm as presented is incomplete. Indeed, cycles or multiple path to a same node may appear in the two computed trees, making them pseudo-trees. There is no guarantee that the provided solution is a pair of valid trees : a tree is defined as an acyclic graph, containing unique paths from the root to each node. The IS computational process only provides a solution in which all target nodes are reached while matching the delay constraint on at least one of each path to each destination.

To ensure the validity of the results, two supplementary steps are necessary : cycles removal, and unique path selection.

Through a Depth First Search (DFS), cycles are identified and eliminated : during the search, if an already visited node is met, it means that the last used edge belongs to a cycle, goes towards the root of the tree and thus should be removed. A new iteration of the DFS rooted to the source is then started. During this removal process, 2-length cycles are processed as special cases. 2-length cycles are defined as cycles between only two nodes.

It has been observed in practice that such cases require specific treatment. Firstly, they are processed as every other cycle and kept in memory for later refinement. Once all cycles have been processed, that is to say 

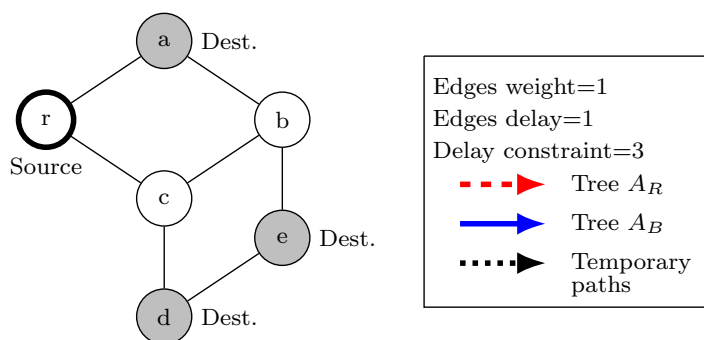

(a) Base graph

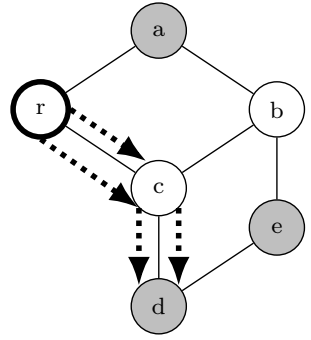

(b) Paths to node $d$

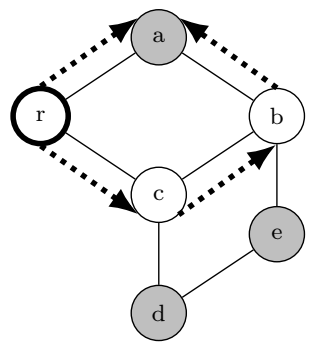

(d) Path to node $a$

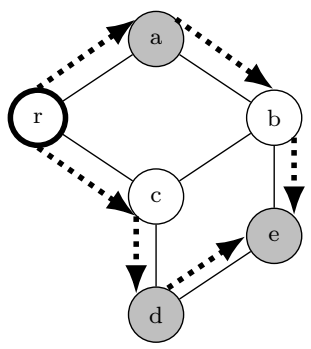

(f) Paths to node $e$

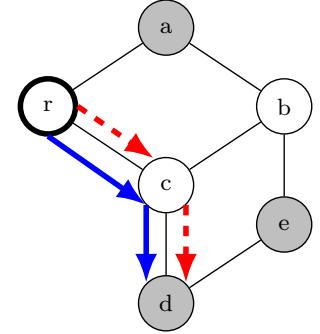

(c) Trees Assignation
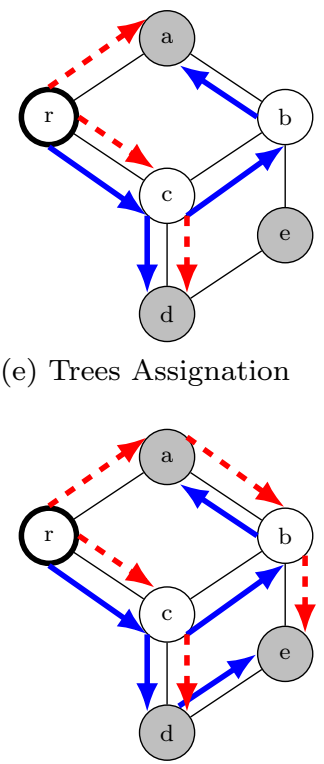

(g) Trees assignation (e) Trees Assignation

Fig. 5 IS Illustration

when the DFS performs complete graph exploration, a new procedure is applied to every 2-length cycle previously met.

Algorithm 3 details the procedure. The principle is the following : both links are examined, and if one of them is required for the tree to be continuous, this link is preserved in the final solution. Otherwise, the shortest-delay path between the two nodes is kept, to ensure that the delay constraint is not violated. Note that this scheme may provide a wrong solution when multiple consecutive 2-length cycle happen in the pseudotree, but this case has rarely been observed in practice.
Once cycles are removed, unique path selection must be performed for the solution to be a valid pair of trees. For any vertex with more than two predecessor in a tree, the shortest-delay path from source is found and only the predecessor matching this path is kept. All other unnecessary edges are removed, that is to say tree branches not leading to a target node. Appendix A, Algorithm 4 details the procedure.

To summarize, to obtain a valid solution from the IS algorithm solution, the following process must be applied :

- DFS rooted at the trees' source is performed to find and break cycles while keeping in memory 2-length cycles met ;

- 2-length cycles are processed for final selection ;

- Multiple paths are eliminated through shortest-delay selection, and useless branches removed.

\subsection{IS algorithm evaluation}

Within TDF network, some multicast services already exploit path diversity. Those services have been used as comparison points to evaluate the performance of the IS algorithm against existing solutions. The network is composed of 928 nodes and 1960 unidirectional links. 43 representative services have been selected for this experiment. The network and affiliated services are subject to trade secret protection and are therefore not further detailed in this paper.

For each existing service, the pair of maximallyindependent trees was designed by experienced network engineers. Within the constrained context of this problem, these human-designed pairs of trees are considered as pseudo-optimums, given that human methods are not able to find better solutions.

The cost function presented in equation (1) is used to evaluate human solution versus the IS-found solution. Results show that in 18 cases the IS-found solution is better, either by bandwidth consumption fine optimization or significant improvement of independence. In 17 cases, it is identical or equivalent to the humanfound solution.

A notable element is that existing services all supply a limited number of destinations (generally inferior to 14), otherwise the problem is to complex to be examined by humans. Therefore, not only the IS algorithm can generally provide better solutions, always faster than a human could, but it can also be applied to problems that were de facto excluded by the operator. 


\section{Architecture's deployment mechanism}

The proposed Seamless Multicast architecture aims to be resilient to multiple failures. Thanks to path diversity, destinations are protected against a single failure. Given maximal path independence, if a failure occurs at least one of the path is still active. But a second failure may occur on this path, and interrupt the stream transportation. In this section, a dynamic scheme for trees' deployment is presented to overcome this drawback. The purpose of this scheme is to ensure that, at any time, a complete pair of trees is deployed on the network, never leaving a destination unsecured.

Traditionally, in case of failure, resilient transport protocols use full path rerouting, segment rerouting or failover components. In the case of multicast protocols, rerouting solutions may be used, for example in the MVPN standard [5]. Nevertheless, this raises some issues : in our case, rerouting implies a loss in the optimality of the solution, and eventually a divergence from the expected transport topology following network's evolution. Failover solutions are also being developed in multicast protocols as in [28].

The objective of the deployment mechanism is to always come back to the assumed optimal state without any impact during transitions. To this end, we propose a successive tree switchover scheme. Indeed, a simple failover mechanism to a new pair of trees would imply an impact. The idea is the following: when the network topology changes, a new pair of tree is computed. If necessary, path rerouting is used to ensure that at least one of the previous trees is reaching all destinations. Then, each previous tree is successively switched to one of the newly computed trees. As a result, there is at any time at least one complete tree supplying the stream to every destination, and in the end the transport topology is optimal. This scheme applies similarly for failures, recovery or network additions. Fig. 6 describes the mechanism and its operation is shown in Fig. 7. Destinations are reached by a pair of trees $A_{R}$ and $A_{B}$. When the link from $d$ to $e$ fails, the $A_{R}$ tree is reconnected, a new pair of trees is computed, the $A_{B}$ tree is switched to a new version while the $A_{R}$ tree sustains the stream, and finally the $A_{R}$ tree is switched to a new version while the $A_{B}$ tree ensures service continuity.

\section{SDN Implementation}

In order to further validate and evaluate the proposed Seamless Multicast architecture, we developed a Proof of Concept SDN controller for experimentation.
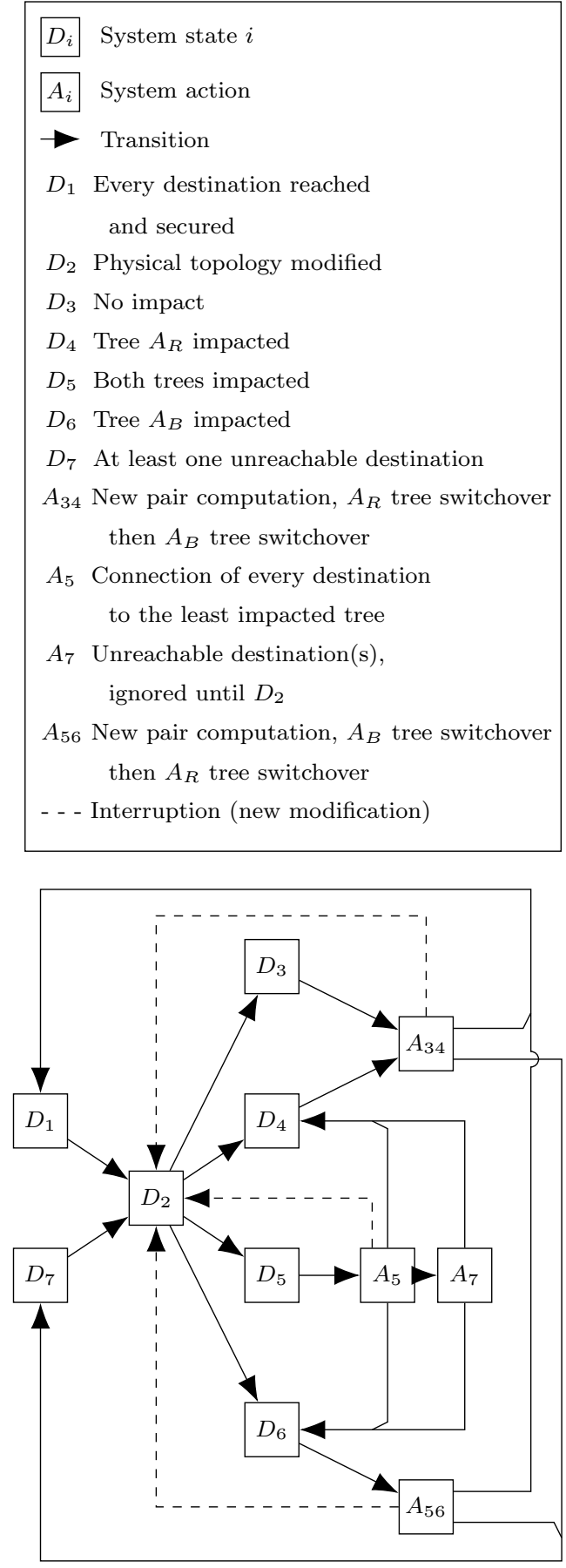

Fig. 6 Deployment mechanism

\subsection{SDN architectures}

SDN is a network architecture aiming at programmable networks. The control plane, traditionally distributed in network nodes, is centralised in the entity called controller. In more advanced designs, the controller can be distributed over a control network. This controller collects data on the network through the management plane, and receives input from the network operator. 

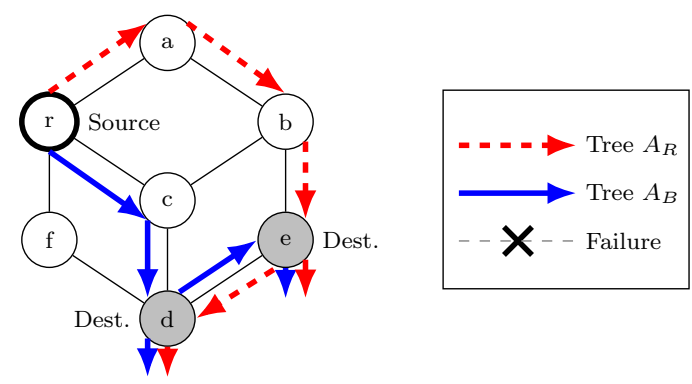

(a) Base graph and deployed trees
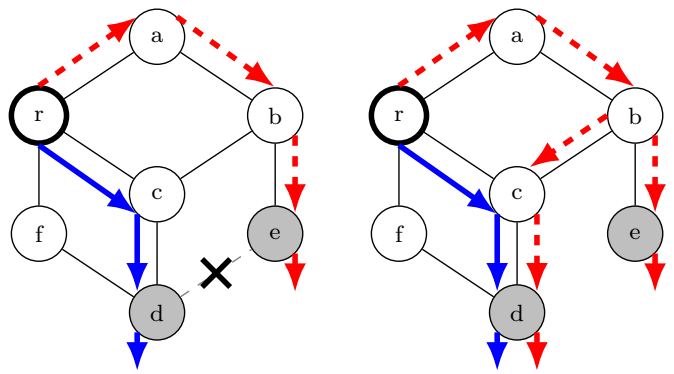

(b) Failure $d$-e $\left(D_{5}\right)$

(c) $A_{R}$ connection $\left(A_{1}\right)$
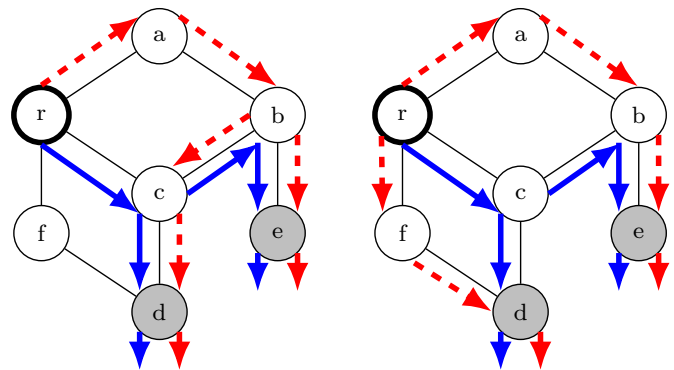

(d) $A_{B}$ switchover $\left(A_{56}\right)$

(e) $A_{R}$ switchover $\left(A_{56}\right)$

Fig. 7 Dynamic switchover illustration

Network nodes are only applying rules defined by the controller's behaviour. The programmability of the network comes from this behaviour, implemented as software in the controller.

The concept of a centralized network architecture is not new, but it recently became more popular and standardized [4]. New technologies are being developed in both the research community and the industry, a good example of which being the Open Networking Foundation.

Due to their flexibility, SDN architectures have been considered for Internet, for example IXP connection [29, 30], but also for Smart Grid energy networks $[31,32]$, VoIP applications [33], or even data-center environment [34].

\subsection{OpenFlow}

The OpenFlow protocol [35], is a technology providing an abstraction layer between the controller and the network nodes.

OpenFlow uses a set of rules called Flows. Each Flow is a combination of a Match, Actions, and statistics. The Match is an identification filter, based on multiple criterion not requiring payload inspection. For example, a Match could be on protocol type, network addresses, incoming port... The Actions belong to a predetermined set, notably including "forwarding", "dropping" or "altering a frame's header". The Actions of a Flow are applied to every data unit filtered by the Match. OpenFlow defines a set of Actions, but not their implementation. As such, vendors retain a competition leverage on performance.

The OpenFlow protocol specifies the communication between network nodes and the controller. The controller can specify Flows, and send them to the nodes. The nodes apply those Flows, and may send to the controller packets that do not match any to ask for new Flows.

Newer versions of the OpenFlow protocol offer more functionalities, notably the Fast Failover Groups from specification 1.1. They are not used in this work, and are not further detailed. Our mechanism only requires elements existing from specification 1.0.

\subsection{Seamless Multicast Controller}

PCE-based architectures are specified in RFC 4655 [36] as architectures in which an entity ensures the computation of network routes with various constraints. This entity called the Path Computation Element (PCE), can be located on a network component or out of the network. An overview of the usage of PCE-based architectures in the literature has been published by Paolucci et al. [37].

This paper's proposed SDN architecture is PCEbased. The PCE is part of the controller, and is based on the IS algorithm presented in Section 4. The logical architecture of our controller is summarized in Fig. 8. A northbound interface connects the network controller to a GUI for operator usage, while a southbound interface enables the controller to communicate with the network elements.

\subsection{Experiments and Results}

For experimental purposes, the controller's implementation is based on the POX controller [38]. It deploys 


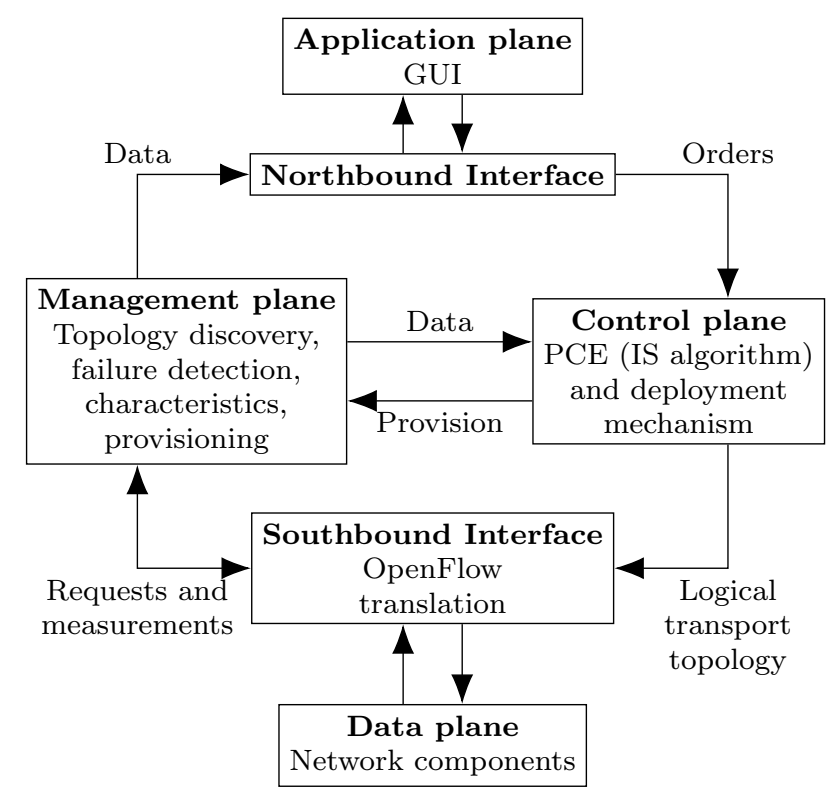

Fig. 8 Implementation architecture

Flows matching on VLAN tagging, forwarding on one or multiple ports. Each VLAN tag is associated with one of the two trees of a pair, and the forwarding ports depend on the path computed by the PCE. As a result, for a given service (i.e. source and set of destinations), there are at most 2 rules per switch.

Validation experiments are led using Mininet [39], a tool for network virtualization. The operating system used for nodes is Open vSwitch [40]. Links' behaviour is emulated using Linux Traffic Control tool.

In order to validate the controller's behaviour, two identical IP streams are forged and sent simultaneously through the network. Each forged packet contains an identifier. Endpoints (i.e. destinations) listen for the two streams, and account for each packet. Consequently, missing or delayed packets are identified, and an elementary Seamless interpretation can be made.

Even though the IP stream are forged and random data are used for padding, the whole infrastructure is real. Mininet provides a virtualization environment, it is not a simulator. This means that even though nodes perform switching faster or slower than real equipment (depending on the physical host), their behaviours are identical. Furthermore, the controller implementation is real (given it is only software), and is connected to the virtual nodes as it would be on a real network.

Various scenarios were tested. Due to space constraint and readability, only one of those will be presented in this paper. The scenario and results are summarized in Fig. 9. The initial state is illustrated in Fig. 9a : the northern node is the source (i.e. where the two streams are emitted), and the two southern nodes in grey $R 1$ and $R 2$ are the destinations, listening for the streams. The controller initially deploys two trees : $A_{R}$ in red dashed lines, and $A_{B}$ in blue full lines. The link $R 1-R 2$ will be the failure location, emulated through Mininet commands.

Fig. 9h illustrates data reception from $R 1$ and $R 2$ for each stream carried by $A_{R}$ and $A_{B}$. The black arrows represent stream synchronisation. In other words, an arrow links two received packets with the same identifier. For ease of reading, each link of the topology has a delay of 1 second.

In the initial state Fig. 9a, both $R_{1}$ and $R_{2}$ receive the two streams. Streams are not synchronized due to different transport path. When the failure occurs in Fig. 9b, each node only receives one stream. The Seamless function ensures the continuity of service, hence no content should be lost. According to the deployment mechanism, the tree $A_{B}$ is rapidly connected in Fig. 9c. Both destinations now receive the stream from the tree $A_{B}$.

Once the new version of the pair of trees has been computed by the PCE, the tree $A_{R}$ is deleted and deployed in its new version in Fig. 9d and Fig. 9e. The reception of the stream from the tree $A_{B}$ on both destination ensures the continuity.

Similarly, the tree $A_{B}$ is also deleted and deployed in Fig. 9f and Fig. 9g, even though in this simple case it is identical to the temporary version.

This example illustrates the deployment mechanism in practice, and shows the valid implementation behaviour.

Even though this implementation is an efficient proof of concept for the Seamless Multicast, multiple implementation issues must be addressed for industrial deployment. The main one is the timing of deployment operations. For example, during dynamic switchover operation, acknowledgement of first tree deployment from the network nodes must be received by the controller before deleting and deploying the second tree. Otherwise, some destinations may be cut off from both streams. However, OpenFlow does not support such acknowledgment. The implementation used in this work uses various timers, tuned according to network characteristics. 


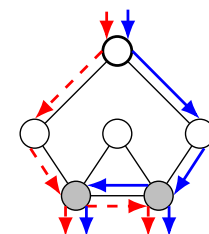

(a) Initial state

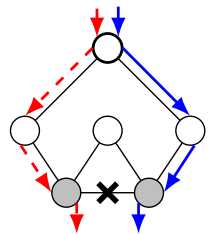

(b) R1 - R2 link failure

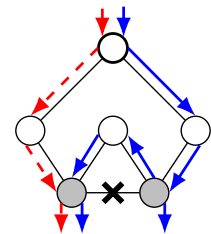

(c) $A_{B}$ connection

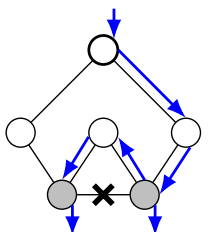

(d) $A_{R}$ deletion

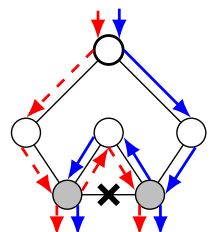

(e) $A_{R}$ deployment

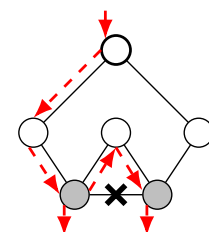

(f) $A_{B}$ deletion

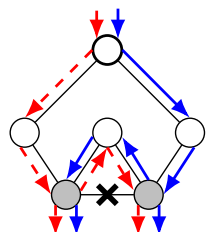

(g) $A_{B}$ deployment

(a) (b) (c) (d) (e) (f) (g)
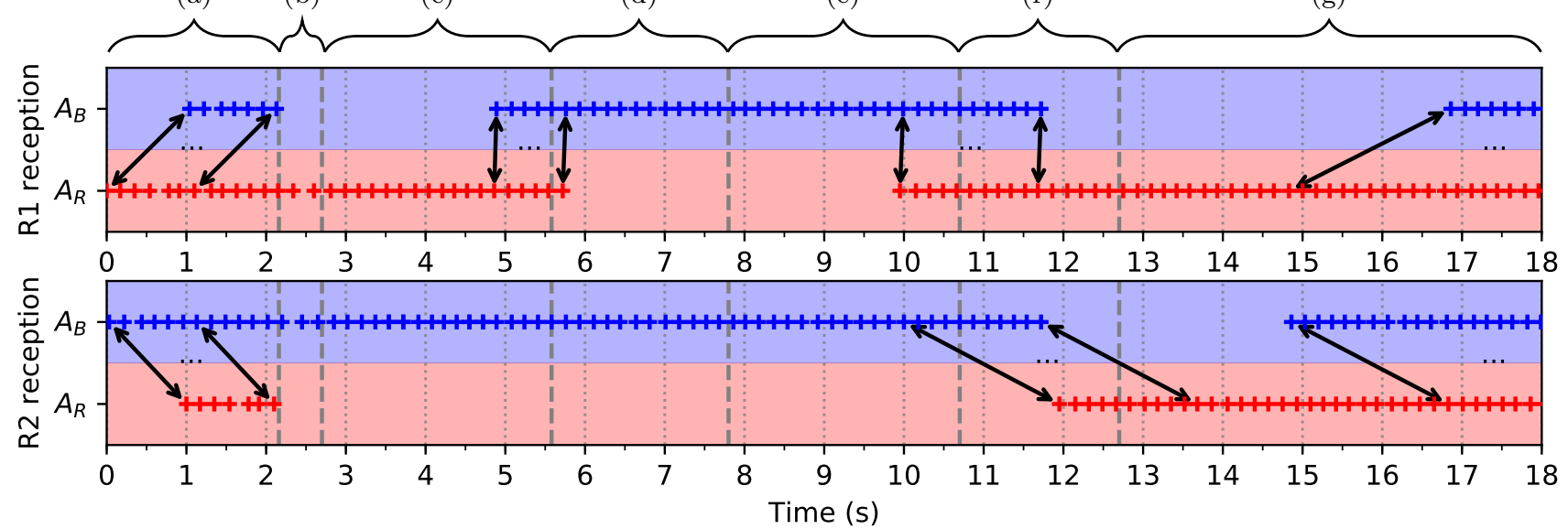

(h) Data reception from R1 (left node) and R2 (right node) of each data stream $A_{R}$ and $A_{B}$

Fig. 9 Sample representative result from experiment led with Proof of Concept Seamless Multicast Controller

\section{Availability and continuity of service enhancement}

The implementation and experimentation provided in Section 6 prove the feasibility of the Seamless Multicast architecture. It also shows that, as intended, for single failure cases, the Seamless Multicast provide a continuity of service that is not expected from the MVPN standard.

To further illustrate the enhancement provided by the Seamless Multicast architecture, we evaluate the availability of the service at each delivery node. To provide an estimation, we will use the data from [41], where the same industrial issue is addressed through traditional network techniques, notably the MVPN standard. As stated, the values presented are representative but not real for trade secret protection.

In [41], Pirlot provides useful but non trivial techniques to accurately estimate the availability of a system using Bayesian networks. Such studies are considered for future works. For this paper's estimation, we use the following reasoning : the unavailability of the Seamless Multicast is equivalent to the simultaneous failures of both trees, or at least failures within a short segment of time corresponding to the $A_{34}$ and $A_{56}$ in- terruptions from Fig. 6. To upper bound a tree's availability, we use the value for a RSTP tree : a single tree, sub-optimal given our constraints, and as it is a spanning tree, it is much more prone to partial failure than one of the trees computed through the IS algorithm.

Let $A_{S M}$ denote the availability of the Seamless Multicast, $A_{A_{R}}$ and $A_{A_{B}}$ the availability of the trees $A_{R}$ and $A_{B}$, and $A_{R S T P}$ the availability of an RSTP tree. Let $P$ denote the respective failure probabilities.

$$
\begin{aligned}
A_{S M} & =1-P_{S M} \\
& =1-P_{A_{R}} * P_{A_{B}} \\
& =1-\left(1-A_{A_{R}}\right) *\left(1-A_{A_{B}}\right) \\
& =1-\left(1-A_{R S T P}\right)^{2}
\end{aligned}
$$

Based on values provided in [41] for what is described as a multicast real case $A_{R S T P}=0.995651019$. Using this value, Table 1 illustrates the improvements provided by the Seamless Multicast. Nearly 18 hours of availability are gained, in other words an improvement of $99 \%$ in availability. Such large numbers are not surprising : the cost of the Seamless Multicast solution is the utilization of twice as much bandwidth, and the implementation of a SDN architecture. 


\begin{tabular}{|c|c|c|}
\hline & MVPN & SM \\
\hline Availability & 0.997858060 & 0.999981086364 \\
\hline $\begin{array}{c}\text { Yearly failure } \\
\text { (h) }\end{array}$ & 18.7633944 & 0.165683449068 \\
\hline $\begin{array}{c}\text { Single failure } \\
\text { impact }\end{array}$ & Always & $\begin{array}{c}\text { Never, except if } \\
\text { failing link is the } \\
\text { single existing } \\
\text { path }\end{array}$ \\
\hline $\begin{array}{c}\text { Overall } \\
\text { Bandwidth } \\
\text { Consumption }\end{array}$ & Simple & Double \\
\hline Network & Traditional & SDN \\
\hline
\end{tabular}

Table 1 MVPN vs Seamless Multicast Comparison

Further works could provide a more complete and precise comparison. For example, the mean time of a failure, the convergence time for MVPN, is a parameter to be studied. For audiovisual content, the length of a impact is a matter of $\mathrm{QoE}$, which requires appropriate measurements.

\section{Conclusion}

In this paper we proposed a new SDN-based architecture called Seamless Multicast, which exploits redundant trees to ensure that a real-time audiovisual stream is transported without interruption from a source to multiple destinations. We proposed a centralized algorithm Iterative SHERPA (IS) to compute maximally edge or node-independent delay-constrained trees. This algorithm is associated with a dynamic switchover mechanism in case of topology change, to guarantee continuity of service and optimisation.

The network programmability provided by SDN architectures allows for the redundancy algorithm and the dynamic switchover mechanism to be deployed in practice. We validated the Seamless Multicast using a Proof of Concept controller implementation over a virtual network. The proposed SDN-based architecture enables continuous transport of audiovisual streams.

Regarding scalability, the proposed IS algorithm is scalable to larger network, and experiments have shown a less than $10 \mathrm{~s}$ computation time on the studied network largest cases. This performance is a major improvement for the industry. The dynamic switchover mechanism itself is only limited by the deployment time of the OpenFlow rules. Even though the number of OpenFlow rules is limited in our proposal, the connection to multiple network nodes for rule installation may be the time-limiting element of the architecture. Henceforth, the Seamless Multicast is scalable to larger networks, and must be tuned to account for control delay.

Finally, even though some technology compatibility issues must be addressed for complete industrial deploy- ment, experiments have shown the expected continuity of service provided by the Seamless Multicast.

\section{References}

1. ITU-T P.10/G.100, "Vocabulary for performance, quality of service and quality of experience," ITU, Tech. Rep., Nov. 2017.

2. ITU-T E.800, "Terms and definitions related to quality of service and network performance including dependability," ITU-T, Tech. Rep., 2009.

3. SMPTE, ST 2022-7:2013: Seamless Protection Switching of SMPTE ST 2022 IP Datagrams. The Society of Motion Picture and Television Engineers, 2013.

4. S. Denazis, E. Haleplidis, J. H. Salim, O. Koufopavlou, D. Meyer, and K. Pentikousis, "RFC 7426 : SoftwareDefined Networking (SDN): Layers and Architecture Terminology," IETF, RFC 7426, 2015.

5. E. C. Rosen and R. Aggarwal, "RFC 6513 : Multicast in MPLS/BGP IP VPNs," IETF, RFC 6513, 2012.

6. S. Pirlot, E. Gnaedinger, F. Lepage, and R. Kopp, "IP/MPLS network modeling using Bayesian networks to improve double failure recovery," in International Conference on Industrial Engineering and Systems Management (IESM), Seville, Spain, 2015, pp. 1155-1160.

7. X. Xiong and T. Chen, "MTM: A Reliable Multiple Trees Multicast for Data Center Network," in 2017 International Conference on Networking, Architecture, and Storage (NAS). Shenzhen, China: IEEE, 2017, pp. 1-7.

8. S. Kubler, J. Robert, J.-P. Georges, and E. Rondeau, "Dual path communications over multiple spanning trees for networked control systems," Engineering Applications of Artificial Intelligence, vol. 25, no. 7, pp. 14601470, 2012.

9. A. Ghannami and C. Shao, "Efficient Fast Recovery Mechanism in Software-Defined Networks: Multipath routing approach," in 2016 11th International Conference for Internet Technology and Secured Transactions (ICITST). Barcelona, Spain: IEEE, 2016, pp. 432-435.

10. M. Vafaei, A. Khademzadeh, and M. A. Pourmina, "QoSaware multi-path video streaming for urban VANETs using ACO algorithm," Telecommunication Systems, vol. 75 , no. 1, pp. 79-96, 2020.

11. S. Islam, N. Muslim, and J. W. Atwood, "A Survey on Multicasting in Software-Defined Networking," IEEE Communications Surveys \& Tutorials, vol. 20, no. 1, pp. 355-387, 2018.

12. L.-H. Yen, M.-H. Wang, S.-Y. Wu, and C.-C. Tseng, "PIM-compliant SDN-enabled IP multicast service," in NOMS 2018 - 2018 IEEE/IFIP Network Operations and Management Symposium. IEEE, 2018, pp. 1-4.

13. D. Kotani, K. Suzuki, and H. Shimonishi, "A Design and Implementation of OpenFlow Controller Handling IP Multicast with Fast Tree Switching," in 2012 IEEE/IPSJ 12th International Symposium on Applications and the Internet. Izmir, Turkey: IEEE, Jul. 2012, pp. 60-67.

14. V. Renganathan Raja, C.-H. Lung, A. Pandey, G.-m. Wei, and A. Srinivasan, "A subtree-based approach to failure detection and protection for multicast in SDN," Frontiers of Information Technology 83 Electronic Engineering, vol. 17 , no. 7 , pp. $682-700$, Jul. 2016.

15. R. Mohammadi, R. Javidan, M. Keshtgari, and R. Akbari, "A novel multicast traffic engineering technique in SDN using TLBO algorithm," Telecommunication Systems, vol. 68, no. 3, pp. 583-592, 2018. 
16. A. Orda and A. Sprintson, "Efficient algorithms for computing disjoint QoS paths," in IEEE INFOCOM 2004, vol. 1, Hong Kong, China, 2004, pp. 727-738.

17. R. Forsati, M. Mahdavi, A. T. Haghighat, and A. Ghariniyat, "An efficient algorithm for bandwidthdelay constrained least cost multicast routing," in Electrical and Computer Engineering, 2008. CCECE 2008. Canadian Conference on. IEEE, 2008, pp. 1641-1646.

18. G. Feng, "Delay constrained multicast routing: What can we learn from an exact approach?" in Global Communications Conference (GLOBECOM), 2012 IEEE. IEEE, 2012, pp. 2809-2814.

19. M. Medard, S. G. Finn, R. A. Barry, and R. G. Gallager, "Redundant Trees for Preplanned Recovery in Arbitrary Vertex-Redundant or Edge-Redundant Graphs," IEEE/ACM Transactions on Networking, vol. 7, no. 5, pp. 641-652, Oct. 1999.

20. Y. Bejerano and P. V. Koppol, "Optimal construction of redundant multicast trees in directed graphs," in INFOCOM 2009, IEEE. IEEE, 2009, pp. 2696-2700.

21. Y. Bejerano, S. Jana, and P. V. Koppol, "Efficient Construction of Directed Redundant Steiner Trees," in Local Computer Networks (LCN), 2012 IEEE 37th Conference on. IEEE, 2012, pp. 119-127.

22. Y. Bejerano and P. V. Koppol, "Link-coloring based scheme for multicast and unicast protection," in High Performance Switching and Routing (HPSR), 2013 IEEE 14th International Conference on. IEEE, 2013, pp. 21-28.

23. R. Widyono, The design and evaluation of routing algorithms for real-time channels. International Computer Science Institute Berkeley, 1994.

24. R. Bellman, "On a routing problem," Quarterly of Applied Mathematics, vol. 16, no. 1, pp. 87-90, Apr. 1958.

25. C. Colombo, F. Lepage, R. Kopp, and E. Gnaedinger, "SHERPA: A SDN Multipath Approach to Eliminate Resilience Impact on Video Streams," in 2018 IEEE 18th International Conference on Communication Technology (ICCT). Chongqing: IEEE, Oct. 2018, pp. 1357-1362.

26. J. W. Suurballe and R. E. Tarjan, "A quick method for finding shortest pairs of disjoint paths," Networks, vol. 14 , no. 2, pp. 325-336, 1984.

27. C. Colombo, F. Lepage, R. Kopp, and E. Gnaedinger, "Two SDN Multi-tree Approaches for Constrained Seamless Multicast," in 2018 IEEE International Conference on Computational Science and Engineering (CSE). Bucharest: IEEE, Oct. 2018, pp. 77-84.

28. T. Morin, R. Kebler, and G. Mirsky, "Multicast VPN fast upstream failover," IETF, Internet-Draft (Work In Progress) 6513, 2020.

29. G. Antichi, I. Castro, M. Chiesa, E. L. Fernandes, R. Lapeyrade, D. Kopp, J. H. Han, M. Bruyere, C. Dietzel, M. Gusat, A. W. Moore, P. Owezarski, S. Uhlig, and M. Canini, "ENDEAVOUR: A Scalable SDN Architecture For Real-World IXPs," IEEE Journal on Selected Areas in Communications, vol. 35, no. 11, pp. 2553-2562, Nov. 2017.

30. M. Bruyere, G. Antichi, E. L. Fernandes, R. Lapeyrade, S. Uhlig, P. Owezarski, A. W. Moore, and I. Castro, "Rethinking IXPs' Architecture in the Age of SDN," IEEE Journal on Selected Areas in Communications, vol. 36, no. 12 , pp. 2667-2674, Dec. 2018.

31. D. Gyllstrom, N. Braga, and J. Kurose, "Recovery from link failures in a Smart Grid communication network using OpenFlow," in 2014 IEEE International Conference on Smart Grid Communications (SmartGridComm). Venice, Italy: IEEE, Nov. 2014, pp. 254-259.
32. N. Dorsch, F. Kurtz, F. Girke, and C. Wietfeld, "Enhanced Fast Failover for Software-Defined Smart Grid Communication Networks," in 2016 IEEE Global Communications Conference (GLOBECOM). Washington, DC, USA: IEEE, Dec. 2016, pp. 1-6.

33. C. A. Hasrouty, V. Autefage, C. Olariu, D. Magoni, and J. Murphy, "SDN-Driven Multicast Streams with Adaptive Bitrates for VoIP Conferences," in 2016 IEEE International Conference on Communications (ICC). Kuala Lumpur, Malaysia: IEEE, May 2016, pp. 1-7.

34. S. Shukla, P. Ranjan, and K. Singh, "MCDC: Multicast routing leveraging SDN for Data Center networks," in 2016 6th International Conference - Cloud System and Big Data Engineering (Confluence). Noida, India: IEEE, Jan. 2016, pp. 585-590.

35. N. McKeown, T. Anderson, H. Balakrishnan, G. Parulkar, L. Peterson, J. Rexford, S. Shenker, and J. Turner, "OpenFlow: Enabling Innovation in Campus Networks," ACM SIGCOMM Computer Communication Review, vol. 38, no. 2, pp. 69-74, 2008.

36. A. Farrel, J.-P. Vasseur, and J. Ash, "RFC 4655 : A path computation element (PCE)-based architecture," IETF, RFC 4655, 2006.

37. F. Paolucci, F. Cugini, A. Giorgetti, N. Sambo, and P. Castoldi, "A Survey on the Path Computation Element (PCE) Architecture," IEEE Communications Surveys $\&$ Tutorials, vol. 15, no. 4, pp. 1819-1841, 2013.

38. "POX Controller." [Online]. Available: https://github.com/noxrepo/pox

39. B. Lantz, B. Heller, and N. McKeown, "A Network in a Laptop: Rapid Prototyping for Software-Defined Networks," in Proceedings of the Ninth ACM SIGCOMM Workshop on Hot Topics in Networks - Hotnets ' 10. Monterey, California: ACM Press, 2010, pp. 1-6.

40. B. Pfaff, J. Pettit, T. Koponen, E. J. Jackson, A. Zhou, J. Rjahalme, J. Gross, A. Wang, J. Stringer, P. Shelar, K. Amidon, and M. Casado, "The Design and Implementation of Open vSwitch," in Proceedings of the 12th USENIX Conference on Networked Systems Design and Implementation. Oakland, CA: USENIX Association, 2015, pp. 117-130.

41. S. Pirlot, "Survivabilité dans les Réseaux de Transport de Vidéo et d'Audio sans Dégradation de la Qualité Perçue par l'Utilisateur," Thèse de doctorat, Université de Lorraine - ED IAEM, Nancy, France, 2016, dirigée par Lepage, Francis. [Online]. Available: http://www.theses.fr/2016LORR0082 


\section{A Complete algorithms}

This appendix presents the complete details of the different algorithms proposed and used in this paper.

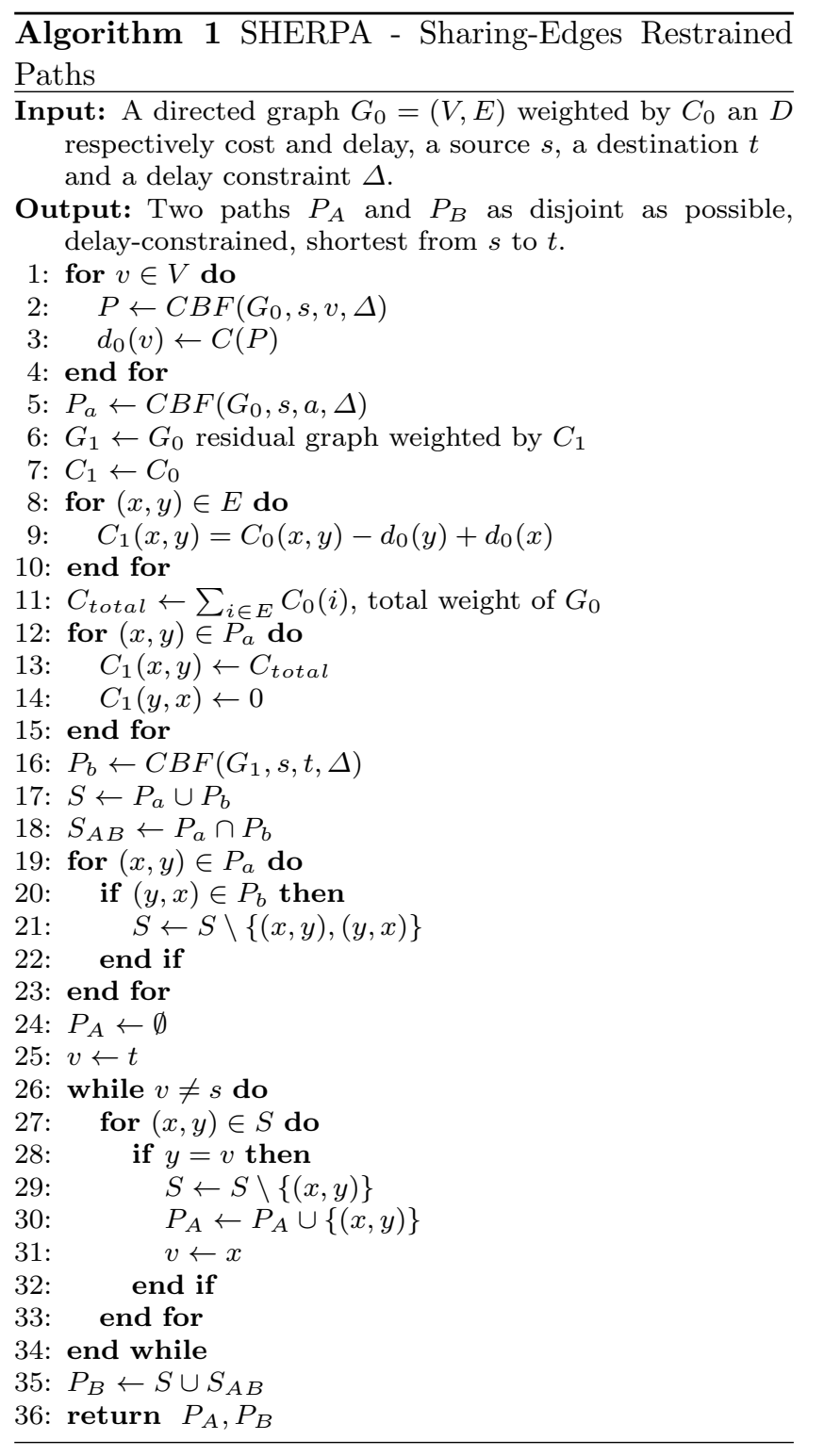

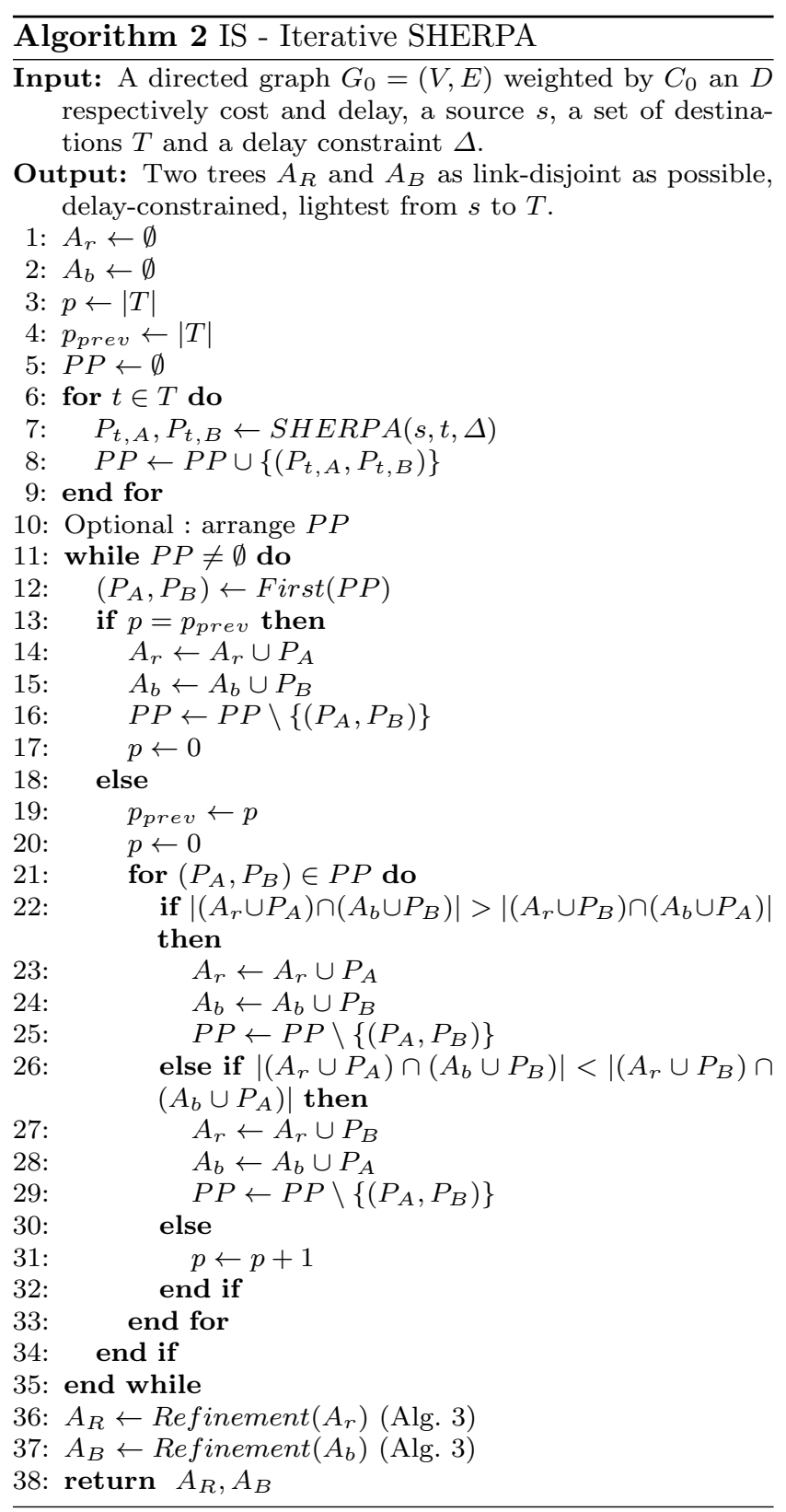



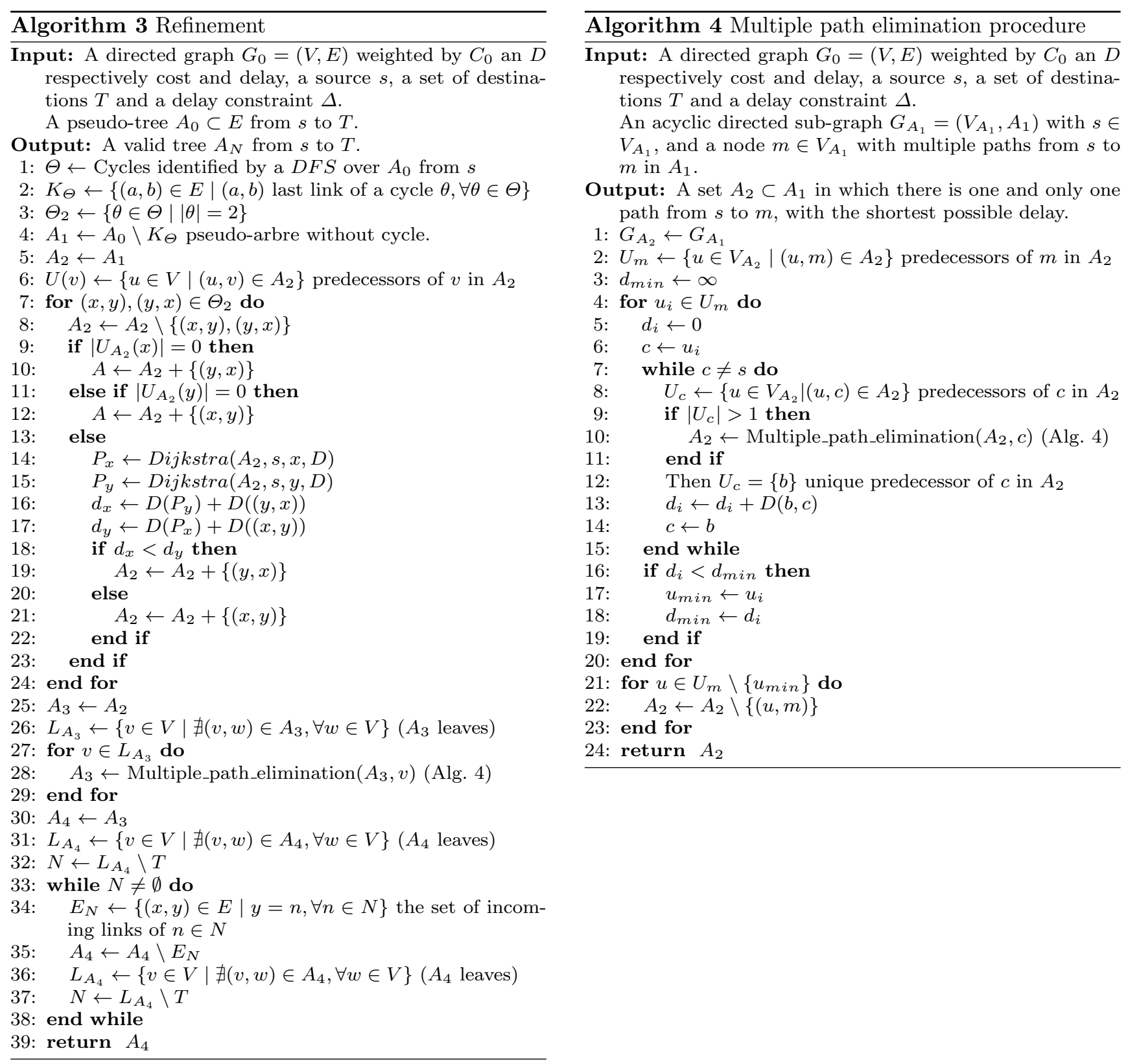